\title{
A Thermal Pressure Inside the Local Bubble, as Revealed by C I Fine-Structure Excitation
}

\author{
Edward B. Jenkins \\ Princeton University Observatory, Princeton, NJ 08544 USA
}

\begin{abstract}
Ultraviolet absorption lines from carbon atoms within a neutral cloud inside or near the edge of the Local Bubble can reveal the thermal pressure of the gas, since the relative populations of the fine-structure levels are determined by a balance between collisional excitation and radiative decay. Features from a C I multiplet appearing in the uv spectrum of the star $\delta$ Cyg $\left(l=79^{\circ}, b=+10^{\circ}\right.$, $d=52 \mathrm{pc}$ ) were observed with the echelle spectrograph of the Goddard High Resolution Spectrograph on HST. An interpretation of absorptions detected from the two lowest levels gives a thermal pressure range $10^{2.7}<p / k<10^{3.7} \mathrm{~cm}^{-3} \mathrm{~K}$.
\end{abstract}

\section{Introductory Remarks}

Observations of the interstellar medium in radio, optical, uv and x-ray spectral regions highlight the existence of tremendous contrasts in density and temperature. We know that even the product of these two quantities, the thermal pressure of the gas, can exhibit significant changes from one region to the next (Jenkins, Jura, \& Loewenstein 1983), indicating that processes that can lead to changes in pressure happen more rapidly than the time needed for the pressures of adjacent, large gas complexes to equalize. Thermal pressures can also be influenced by an interplay with other forms of pressure, such as turbulence, cosmic rays and magnetic fields (Kulkarni \& Heiles 1988). Against this chaotic background, it is clear that we must explicitly measure pressures in specific contexts of interest and not take overall averages determined from elsewhere. Thus, in understanding the fundamental nature of the Local Bubble, our interpretations must rest upon the properties of gas that resides within its perimeter, or perhaps just beyond it. While the thermal pressure $n k T$ is only one of several possible forms of pressure, it is a measurable physical property that must be reconciled with various plausible theoretical alternatives for the evolution and present state of the Local Bubble.

\section{Method of Measurement}

For any cold, mostly neutral cloud in front of an early-type star, we can observe absorption features from neutral carbon, even though the dominant ionization stage is the singly ionized form. The ground state of $\mathrm{C} \mathrm{I}$ is split into 
three fine-structure levels, representing different $J$ quantum states. The upper two levels have energy separations $E / k$ from the ground level that are comparable to typical interstellar kinetic temperatures, and they are populated by collisional excitations by other gas constituents (atoms and electrons). ${ }^{1}$ Their relative occupations are governed by an equilibrium between these collisions that scale with local density and a constant rate of depopulation via radiative decays in the infrared.

A formalism introduced by Jenkins \& Shaya (1979) for expressing the relative populations,

$$
f_{1}=\frac{N\left(\mathrm{C} \mathrm{I}^{*}\right)}{N\left(\mathrm{C} \mathrm{I}_{\text {total }}\right)} \quad \text { and } \quad f_{2}=\frac{N\left(\mathrm{C} \mathrm{I}^{* *}\right)}{N\left(\mathrm{C} \mathrm{I}_{\text {total }}\right)}
$$

can be used to interpret the results in terms of various temperatures and pressures through a diagram presented in their Figure 6. $N\left(\mathrm{C} \mathrm{I}^{*}\right)$ is the column density of carbon atoms in the first excited level $\left({ }^{3} P_{1}\right), N\left(\mathrm{C} \mathrm{I}^{* *}\right)$ that of the second excited level $\left({ }^{3} P_{2}\right)$, and $N\left(\mathrm{C} \mathrm{I}_{\text {total }}\right)$ applies to the sum of all three levels. (A particular advantage of this representation is that one can use a simple geometrical interpretation to understand the result from a superposition of two regions with different conditions, as explained by Jenkins \& Shaya, but this complication will not apply to the case studied here.)

\section{Observation}

The investigation of pressures for the Local Bubble using C I absorption features was the objective of a Cycle 6 Guest Observer program with HST. We consider here the results for one star in this small survey, $\delta$ Cyg, a B9.5III star with a $\mathrm{V}$ magnitude of 2.92 and an apparent rotation velocity $v \sin i=$ $149 \mathrm{~km} \mathrm{~s}^{-1}$ (Hoffleit \& Jaschek 1982). It is at a distance of $52 \mathrm{pc}$ (Hipparcos parallax) and is located in the direction $l=79^{\circ}, b=+10^{\circ}$. In this general direction, appreciable concentrations of $\mathrm{H} \mathrm{I}$ are found at far greater distances (Paresce 1984), but some stars in the general vicinity of $\delta$ Cyg do show some Ca II absorption (Vallerga, et al. 1993). Fig. 1 shows the spectrum that was recorded by the echelle spectrograph in GHRS $(\lambda / \Delta \lambda=80,000)$, using the large, $2^{\prime \prime}$ aperture with COSTAR in front.

Since C I was detected only in this one multiplet, we can not derive $N(\mathrm{C} \mathrm{I})$ from a curve of growth, and therefore we must determine the line saturations indirectly. Absorption lines of $\mathrm{Na} I$ recorded at visible wavelengths should be good analogues to those of C I, since they too originate from a very mildly depleted element in an ionization stage below the preferred one. Very high resolution spectra of $\delta \mathrm{Cyg}$ indicate that $b(\mathrm{NaI})=0.43 \mathrm{~km} \mathrm{~s}^{-1}$ (Blades,

${ }^{1}$ If the atoms are near a star that emits a large flux of uv photons, optical pumping of the levels can also be important. See Fig. 6b of Jenkins \& Shaya (1979). 


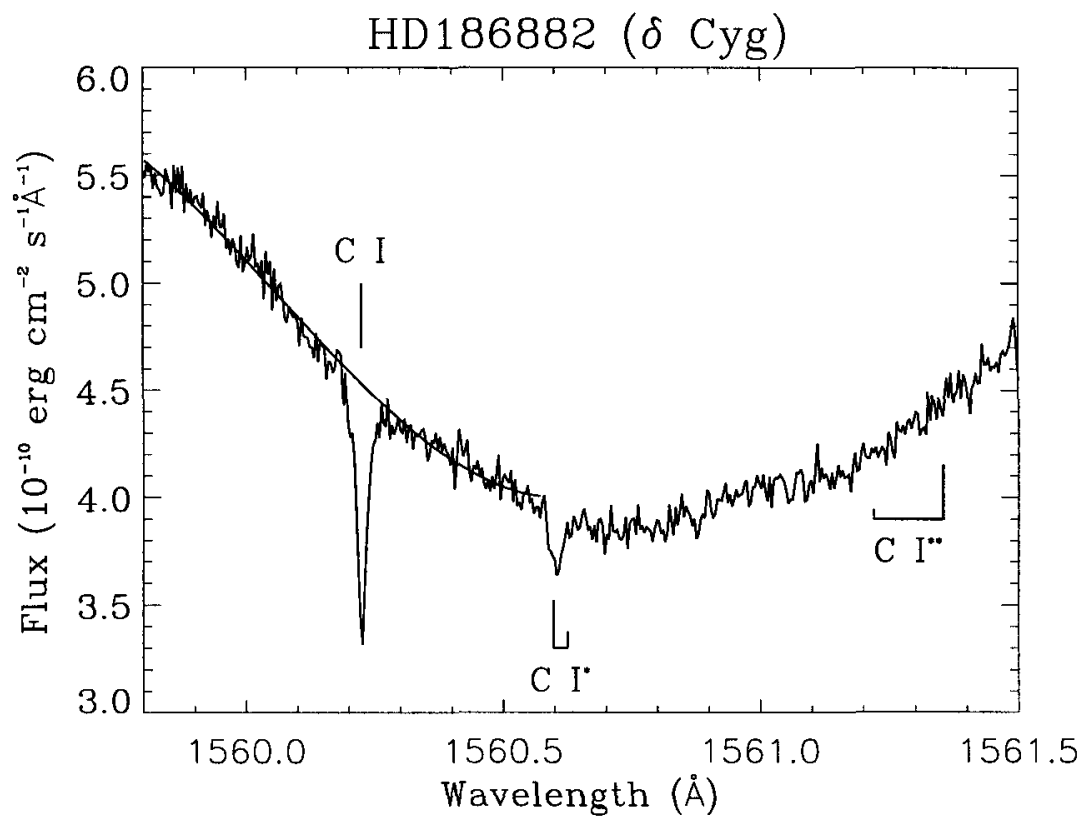

Fig. 1. The spectrum of $\delta$ Cyg covering the $1560 \AA$ multiplet of $C$ I. The positions of absorptions out of the 3 fine-structure levels are shown with labels for C I, C I*, and $\mathrm{C} \mathrm{I}^{* *}$. The lengths of the vertical markers are proportional to the lines' $\mathrm{f}$-values. Where there are two transitions from the same level, the vertical markers are joined by a horizontal line. The smooth line shows the adopted continuum level for the strong C I line.

Wynne-Jones, \& Wayte 1980; Welty, Hobbs, \& Kulkarni 1994), so we expect that $b(\mathrm{CI})$ is somewhere between 0.43 and $0.60 \mathrm{~km} \mathrm{~s}^{-1}$, depending on whether the broadening is primarily turbulent or thermal. ${ }^{2}$ If it is turbulent, the temperature could be arbitrarily low, while for pure thermal broadening $b(\mathrm{Na} \mathrm{I})$ leads to $T=250 \mathrm{~K}$. The table below summarizes the outcomes for these two extremes for the permissible ranges of $f_{1}$. Upper limits for $f_{2}$ do not provide useful constraints. For the physical state of the cloud in front of $\delta$ Cyg (i.e., mostly neutral, cool gas) the fine structure levels are predom-

${ }^{2}$ From a determination that $b(\mathrm{Ca} I \mathrm{II})=0.47 \mathrm{~km} \mathrm{~s}^{-1}$ toward $\delta$ Cyg (Welty, Morton, $\&$ Hobbs 1996), we might be tempted to conclude that the broadening must be mostly turbulent and the temperature is very low, since $\mathrm{Ca}$ atoms are almost twice as heavy as $\mathrm{Na}$ atoms. Unfortunately, we could be fooled by a general tendency for Ca II lines to appear broader than lines of $\mathrm{Na} I$ (see Fig. 7 of Welty et al.), an effect that could cancel the $m^{-1 / 2}$ scaling for pure thermal Doppler broadening. Our HST measurement does not have enough resolution to determine $b$ (C I) from the apparent profile shape (Robinson, et al. 1995). 
inantly excited by collisions with $\mathrm{H} \mathrm{I}$; a fractional ionization greater than about $1 \%$ would be needed before electrons could have much effect on the value of $f_{1}$ (Keenan 1989).

\begin{tabular}{ccr|rr|rr} 
Source of & $b(\mathrm{C} \mathrm{I})$ & $T$ & \multicolumn{2}{|c}{$f_{1}$} & \multicolumn{2}{c}{$\log p / k$} \\
Line Broadening & $\left(\mathrm{km} \mathrm{s}^{-1}\right)$ & $(\mathrm{K})$ & l.l. & u.l. & 1.1. & u.l. \\
\hline pure thermal & 0.60 & 250 & 0.121 & 0.185 & 3.45 & 3.70 \\
pure turbulent & 0.43 & 40 & 0.065 & 0.128 & 2.70 & 3.10 \\
\hline
\end{tabular}

The allowed range $10^{2.7}<p / k<10^{3.7} \mathrm{~cm}^{-3} \mathrm{~K}$ for the thermal pressure of the cold cloud toward $\delta$ Cyg is well centered on the estimated thermal pressure of another cloud in the Local Bubble, namely, the partly ionized, warm medium that surrounds the Sun (Wood \& Linsky 1997; Lallement, 1997). However, it is significantly lower than the general estimate of $1-2 \times$ $10^{4} \mathrm{~cm}^{-3} \mathrm{~K}$ for hot gas in the Local Bubble (Cox \& Reynolds 1987) or the measurement of Bowyer, et al. (1995).

Acknowledgements. Support for this work was provided by NASA through grant number GO-06415.01-95A from the Space Telescope Science Institute, which is operated by the Association of Universities for Research in Astronomy, Inc., under NASA contract NAS5-26555.

\section{References}

Blades, J. C., Wynne-Jones, I., \& Wayte, R. C. 1980: MNRAS, 193, 849

Bowyer, S., Lieu, R., Sidher, S. D., Lampton, M., \& Knude, J. 1995: Nature, 375, 212

Cox, D. P., \& Reynolds, R. J. 1987: Ann. Rev. A\&A, 25, 303

Hoffleit, D., \& Jaschek, C. 1982: The Bright Star Catalogue, (New Haven: Yale U. Obs.)

Jenkins, E. B., Jura, M., \& Loewenstein, M. 1983: ApJ, 270, 88

Jenkins, E. B., \& Shaya, E. J. 1979: ApJ, 231, 55

Keenan, F. P. 1989: ApJ, 339, 591

Kulkarni, S. R., \& Heiles, C. 1988: in Galactic and Extragalactic Radio Astronomy, ed. G. L. Verschuur \& K. I. Kellermann (Berlin: Springer), p. 95

Lallement, R. 1997: these proceedings

Paresce, F. 1984: Astron. J., 89, 1022

Robinson, R. D. 1995: in Calibrating Hubble Space Telescope: Post Servicing Mission, ed. A. Koratkar \& C. Leitherer (Baltimore: Space Telescope Science Inst.), p. 193

Vallerga, J. V., Vedder, P. W., Craig, N., and Welsh, B. Y. 1993: ApJ, 411, 729

Welty, D. E., Hobbs, L. M., \& Kulkarni, V. P. 1994: ApJ, 436, 152

Welty, D. E., Morton, D. C., \& Hobbs, L. M. 1996: ApJS, 106, 533

Wood, B. E. \& Linsky, J. L. 1997: ApJL, 474, L39 